\title{
ROJ
}

\section{Long-term outcomes after salvage radiotherapy for postoperative locoregionally recurrent non-small-cell lung cancer}

\author{
Eunji Kim, MD', Changhoon Song, MD, PhD'1, Mi Young Kim, MD² Jae-Sung Kim, MD, PhD' \\ 'Department of Radiation Oncology, Seoul National University College of Medicine, Seoul; \\ ${ }^{2}$ Department of Radiation Oncology, Kyungpook National University Medical Center, Daegu, Korea
}

Purpose: The outcomes and toxicities of locoregionally recurrent non-small-cell lung cancer (NSCLC) patients treated with curative radiotherapy were evaluated in the modern era.

Materials and Methods: Fifty-seven patients receiving radical radiotherapy for locoregionally recurrent NSCLC without distant metastasis after surgery from 2004 to 2014 were reviewed. Forty-two patients were treated with concurrent chemoradiotherapy (CCRT), and 15 patients with radiotherapy alone. The median radiation dose was $66 \mathrm{~Gy}$ (range, 45 to 70 Gy). Lung function change after radiotherapy was evaluated by comparing pulmonary function tests before and at 1, 6, and 12 months after radiotherapy. Results: Median follow-up was 53.6 months (range, 12.0 to 107.5 months) among the survivors. The median overall survival (OS) and progression-free survival (PFS) were 54.8 months (range, 3.0 to 116.9 months) and 12.2 months (range, 0.8 to 100.2 months), respectively. Multivariate analyses revealed that single locoregional recurrence focus and use of concurrent chemotherapy were significant prognostic factors for OS ( $p=0.048$ and $p=0.001$, respectively) and PFS ( $p=0.002$ and $p=0.026$, respectively). There was no significant change in predicted forced expiratory volume in one second after radiotherapy. Although diffusing lung capacity for carbon monoxide decreased significantly at 1 month after radiotherapy $(p<0.001)$, it recovered to pretreatment levels within 12 months. Acute grade 3 radiation pneumonitis and esophagitis were observed in 3 and 2 patients, respectively. There was no chronic complication observed in all patients.

Conclusion: Salvage radiotherapy showed good survival outcomes without severe complications in postoperative locoregionally recurrent NSCLC patients. A single locoregional recurrent focus and the use of CCRT chemotherapy were associated with improved survival. CCRT should be considered as a salvage treatment in patients with good prognostic factors.

Keywords: Non-small-cell lung carcinoma, Local recurrence, Radiotherapy

\section{Introduction}

Lung cancer is the leading cause of cancer death accounting for $22.6 \%$, and the incidence is the 4 th in cancer statistics in
Korea [1]. The age-standardized incidence rate of lung cancer in men has decreased, while that in women has increased from 1999 to 2013. Although surgical resection is the treatment of choice for early stage non-small-cell lung cancer (NSCLC)

Received 28 August 2016, Revised 26 October 2016, Accepted 9 November 2016.

Correspondence: Jae-Sung Kim, MD, PhD, Department of Radiation Oncology, Seoul National University Bundang Hospital, 82 Gumi-ro 173beon-gil, Bundang-gu, Seongnam 13620, Korea. Tel: +82-31-787-7652, Fax: +82-31-787-4019, E-mail: jskim@ snubh.org

(c) This is an Open Access article distributed under the terms of the Creative Commons Attribution Non-Commercial License (http://creativecommons.org/ licenses/by-nc/4.0/) which permits unrestricted non-commercial use, distribution, and reproduction in any medium, provided the original work is properly cited.

www.e-roj.org 
patients, recurrence rates after surgery have been reported to be from 28\% to 60\%, and postrecurrence survival was poor [2-4]. The standard treatment for locoregionally recurrent NSCLC is still controversial. Repeated operation, radiation therapy, and concurrent chemoradiotherapy (CCRT) are considered for salvage local therapy. Even with the favorable survival outcomes, salvage reoperation is limited in selected patients [5]. Alternatively, patients could be treated with salvage radiotherapy with or without chemotherapy. The recent National Comprehensive Cancer Network guideline recommends CCRT in case of mediastinal lymph node recurrence and superior vena cava obstruction [6].

Several researchers have already evaluated treatment outcomes of recurrent NSCLC treated with salvage radiotherapy [7-13]. They reported the median survival ranging from 14 to 31 months. The survival of patients treated with salvage radiotherapy after recurrence was comparable to that of patients initially treated with radical radiotherapy [9]. Furthermore, investigators demonstrated that CCRT could be a promising treatment modality for recurrent NSCLC $[11,12]$. However, these studies were performed with relatively short follow-up period. Thus, these results might be exaggerated.

On the other hand, radiotherapy to the thorax can cause side effects. Some patients can experience a significant decrease in pulmonary function after curative radiotherapy, and cannot recover in long-term follow-up [14]. In case of postoperative setting, these complications might be worse. According to a trial evaluating postoperative radiotherapy, high incidence of death, cardiac and respiratory complications were observed [15]. Because previous studies have focused only on the treatment outcome of salvage radiotherapy, little information is available regarding toxicities of these patients.

This study retrospectively analyzed the survival outcomes and complications of patients with locoregionally recurrent NSCLC who were treated with salvage radiotherapy for a longterm follow-up period. We observed the changes of values of pulmonary functions tests after radiotherapy quantitatively.

\section{Materials and Methods}

\section{Patients}

This study was performed after the approval of the Institutional Ethical Review Board. We retrospectively reviewed the medical records of patients with recurrent NSCLC treated with salvage radiotherapy between 2004 and 2014. The inclusion criteria were as follows: (1) initially resected NSCLC under curative intention without adjuvant radiotherapy; (2) clinically or pathologically confirmed locoregional recurrence; (3) no evidence of distant metastasis; and (4) definitive aim radiotherapy for recurrent NSCLC. Locoregional recurrence was defined as a recurrence in contiguous sites to primary tumor or regional lymph nodal area. We identified 57 patients who fulfilled the inclusion criteria. When the recurrence was suspected, all patients underwent chest computed tomography (CT), 18-fluoro-deoxyglucose positron emission tomographyCT (PET-CT) and chest X-ray. Pathologic confirmation of the recurrence was done in 28 patients (49.1\%). All patients were re-staged as if the patients had been newly diagnosed according to the 7th edition of the American Joint Committee on Cancer Staging.

\section{Treatment}

Planning CT was obtained in a supine position with 3- or 4-mm slice thickness. Gross tumor volume (GTV) included recurrent tumor sites only regardless of primary location. Bronchial stump or ipsilateral hilar lymph nodes were not routinely included in the delineation of the clinical target volume (CTV). Lung parenchymal lesion was delineated in a pulmonary window, and mediastinal or hilar/interlobar lymph nodes were delineated in a mediastinal setting. CTV was defined by a 5 - to 8-mm expansion from the GTV, and planning target volume was created by $10 \mathrm{~mm}$ margin from CTV. Radiotherapy was administered with 6-15 MV photons from linear accelerator with 3-dimensional conformal technique in all patients. The total dose was 45 to $70 \mathrm{~Gy}$, delivered in daily fractions of 1.8 to 3.0 Gy. Thirty-one patients (54.4\%) received 66 Gy in 33 fractions. Two patients received $2.5 \mathrm{~Gy}$ per fraction (65 Gy in 1 patient and $60 \mathrm{~Gy}$ in 1); 5 patients received 3 Gy per fraction (60 Gy in 2 patients, 54 Gy in 1,51 Gy in 1, and 45 Gy in 1). Median biologically equivalent dose (BED, assuming $\alpha / \beta=10$ ) was 79.2 Gy (range, 58.5 to 84 Gy). Forty-two patients (73.7\%) received concurrent chemotherapy in addition to radiotherapy. Chemotherapy regimen used in the treatment was weekly paclitaxel $50 \mathrm{mg} / \mathrm{m}^{2}$ combined with cisplatin $25 \mathrm{mg} / \mathrm{m}^{2}$ ( $\mathrm{n}=$ 40) or triweekly etoposide $100 \mathrm{mg} / \mathrm{m}^{2}$ combined with cisplatin $70 \mathrm{mg} / \mathrm{m}^{2}(\mathrm{n}=2)$.

\section{Follow-up}

Patients were regularly followed up with physical examination, blood tests, pulmonary function tests (PFTs) and chest CT. Radiologic tumor response to radiotherapy was evaluated using response evaluation criteria in solid tumors (RECIST) criteria by chest CT taken 1 month after radiotherapy. Locoregional recurrence was defined as recurrence in the 
primary tumor and a regional lymph nodal area. We also checked whether radiation in-field recurrence occurred or not. Distant metastasis was defined as recurrence in the ipsilateral different lobe, the contralateral lung, pleural nodules, malignant effusion, or extra-thoracic organs. Acute and late treatment related toxicities were evaluated by the physician according to the National Cancer Institute's Common Terminology Criteria for Adverse Events (CTCAE) ver. 4.03. Complication occurred within 3 months from the start of radiotherapy was defined as acute complication, and after 3 months was as chronic complication. Patients performed PFTs including forced expiratory volume in 1 second (FEV1) and diffusing capacity of the lung for carbon monoxide (DLCO) at baseline and at 1, 6, and 12 months after radiotherapy. The results were also evaluated on the basis of each study's percentage of predicted of normal values adjusted for age, gender, and height.

\section{Statistical analysis}

Initial disease-free interval was calculated from the date of operation to the diagnosis of recurrence. Locoregional recurrence-free survival (LRFS), distant metastasis-free survival (DMFS), progression-free survival (PFS), and overall survival (OS) were calculated from the end day of radiotherapy to the event. Survival was estimated with the Kaplan-Meier method. The logrank test and the Cox proportional hazard model were used for univariate and multivariate analyses, respectively. Multivariate analysis was performed on variables with a $p$-value $<0.1$ in univariate analysis. Baseline and postradiotherapy PFT values were presented as mean \pm standard deviation (SD) and analyzed using paired t-test. Factors with a $p$-value of less than 0.05 were regarded as statistically significant. All analyses were performed with PASW ver. 23.0 (SPSS Inc., Chicago, IL, USA).

\section{Results}

\section{Patients and tumor characteristics}

Patient characteristics are shown in Table 1. The median age at the recurrence was 67 years (range, 34 to 81 years). There were 44 men and 13 women. Forty-eight patients (84.2\%) showed an Eastern Cooperative Oncology Group performance status (ECOG PS) 0 or 1 at the diagnosis of recurrence. Initial pathologic stage according to the TNM 7th edition was stage I in 25 patients (43.9\%), stage II in 17 patients (29.8\%), and stage III in 15 patients (26.3\%). Initially, 47 patients (82.5\%) received lobectomy. Adenocarcinoma and squamous cell carcinoma accounted for $40.4 \%$ each. Adjuvant chemotherapy was administered to 40 patients (70.2\%). Median disease-

Table 1. Patients' characteristics

\begin{tabular}{|c|c|}
\hline Variable & No. of patients (\%) \\
\hline Age (yr) & $67(34-81)$ \\
\hline \multicolumn{2}{|l|}{ Gender } \\
\hline Man & $44(77.2)$ \\
\hline Woman & $13(22.8)$ \\
\hline \multicolumn{2}{|l|}{ ECOG performance status } \\
\hline $0-1$ & $48(84.2)$ \\
\hline 2 & $9(15.8)$ \\
\hline \multicolumn{2}{|l|}{ Initial pathologic stage } \\
\hline I & $25(43.9)$ \\
\hline II & $17(29.8)$ \\
\hline III & $15(26.3)$ \\
\hline \multicolumn{2}{|l|}{ Initial operation } \\
\hline Pneumonectomy & $5(8.8)$ \\
\hline Bilobectomy & $2(3.5)$ \\
\hline Lobectomy & $47(82.5)$ \\
\hline Wedge resection & $3(5.3)$ \\
\hline \multicolumn{2}{|l|}{ Histology } \\
\hline Adenocarcinoma & $23(40.4)$ \\
\hline Squamous cell carcinoma & $23(40.4)$ \\
\hline Others & $11(19.3)$ \\
\hline Disease-free interval (mo) & $13.6(1.4-57.4)$ \\
\hline \multicolumn{2}{|l|}{ Recurrence stage } \\
\hline I, II & $18(31.6)$ \\
\hline III & $39(68.4)$ \\
\hline \multicolumn{2}{|l|}{ Recurrence site } \\
\hline Superior mediastinal LN & $25(43.9)$ \\
\hline Hilar/interlobar LN & $15(26.3)$ \\
\hline Lung parenchyma & $15(26.3)$ \\
\hline Supraclavicular LN & $9(15.8)$ \\
\hline Inferior mediastinal LN & $9(15.8)$ \\
\hline Bronchial stump & $6(10.5)$ \\
\hline Aortic LN & $5(8.8)$ \\
\hline \multicolumn{2}{|c|}{ No. of locoregional recurrent foci } \\
\hline 1 & 37 (64.9) \\
\hline 2 & $13(22.8)$ \\
\hline 3 & 7 (12.3) \\
\hline \multicolumn{2}{|l|}{ Salvage treatment } \\
\hline CCRT & 42 (73.7) \\
\hline RT alone & 15 (26.3) \\
\hline $\operatorname{GTV}\left(\mathrm{cm}^{3}\right)$ & $39.9(1.0-283.4)$ \\
\hline BED $\left(\mathrm{Gy}_{10}\right)$ & 79.2 (58.5-84) \\
\hline \multicolumn{2}{|l|}{ Fractional dose (cGy) } \\
\hline 180, 200 & $50(87.7)$ \\
\hline 250,300 & 7 (12.3) \\
\hline
\end{tabular}

Values are presented as median (range) or number (\%). ECOG, Eastern Cooperative Oncology Group; LN, Iymph node; CCRT, concurrent chemoradiotherapy; RT, radiotherapy; GTV, gross tumor volume; BED, biologically equivalent dose. 
Table 2. Patterns of failure

\begin{tabular}{lc}
\hline \multicolumn{1}{c}{ Variable } & No. of patients (\%) \\
\hline Recurrence & \\
Yes & $39(68.4)$ \\
No & $18(31.6)$ \\
Locoregional recurrence & $17(29.8)$ \\
Isolated in-field recurrence & $5(8.8)$ \\
Isolated out-field recurrence & $3(5.3)$ \\
Locoregional + distant recurrence & $9(15.8)$ \\
Distant recurrence & $31(54.4)$ \\
Isolated distant recurrence & $22(38.6)$ \\
Locoregional + distant recurrence & $9(15.8)$ \\
\hline
\end{tabular}

free interval was 13.6 months (range, 1.4 to 57.4 months). In our cohort, the most commonly recurred site after surgery was superior mediastinal lymph nodal area ( $n=25,43.9 \%)$. As regard to the number of locoregional recurrent foci, single locoregional focus was in 37 patients (64.9\%). The median volume of GTV was $39.9 \mathrm{~cm}^{3}$ (range, 1.0 to $283.4 \mathrm{~cm}^{3}$ ). There was significant difference in patients' age, PS, and rate of lung parenchymal recurrence between CCRT group and radiotherapy alone group ( $p<0.001, p=0.034$, and $p=0.001$, respectively).

\section{Treatment outcomes and failure patterns}

The radiologic tumor response after radiotherapy was as follows: 6 patients (10.5\%) achieved a complete regression, 39 patients (68.4\%) achieved a partial regression, 10 patients $(17.5 \%)$ had a stable disease, and 2 patients (3.5\%) had progressive disease. The median follow-up time was 24 months (range, 3.0 to 119.8 months) for all patients, and 53.6 months (range, 12.0 to 107.5 months) for surviving patients. Treatment failure occurred in 39 patients (68.4\%). Detailed patterns of failure are shown in Table 2. Seventeen patients (29.8\%) failed locoregionally, and 5 of them showed an isolated in-field recurrence. Thirty-one patients (54.4\%) failed systemically. The most common site of distant metastasis was a contralateral lung, followed by pleura, brain, bone, and liver.

\section{Survival and prognostic factors}

The median OS and PFS times were 54.8 months (range, 3.0 to 116.9 months) and 12.2 months (range, 0.8 to 100.2 months), respectively. The 2-year OS, PFS, LRFS, and DMFS rates were $62.4 \%, 33.6 \%, 70.9 \%$, and 47.4\%, respectively. The 5-year OS, PFS, LRFS, and DMFS rates were 48.5\%, 20.5\%, 63.8\%, and 33.5\%, respectively (Fig. 1).

Table 3 provides the results from univariate and multivariate analyses for OS. On univariate analysis, age, and concurrent chemotherapy were significant prognostic factors for OS Multivariate analysis of revealed that multiple locoregional recurrent foci $(p=0.048)$ and radiotherapy alone $(p=0.001)$ were adverse prognostic factors for OS. Fig. 2 illustrates the Kaplan-Meier curves according to the use of concurrent chemotherapy and the number of recurrence foci. Median OS were 86 months in patients receiving CCRT and 20 months in those receiving radiotherapy alone ( $p=0.001)$ (Fig. $2 A)$. In addition, median OS were 86 months in patients with a single recurrent focus and 21 months in those with multiple recurrent foci ( $p=0.085$ ) (Fig. 2B).

As regard to PFS, univariate analysis determined that age and the number of recurrent foci were significant prognostic factors (Table 4). On multivariate analysis, single locoregional recurrent focus $(p=0.002)$ and the use of concurrent chemotherapy ( $p=0.026)$ were independently associated with improved PFS. Seven patients (12.3\%) were still alive without

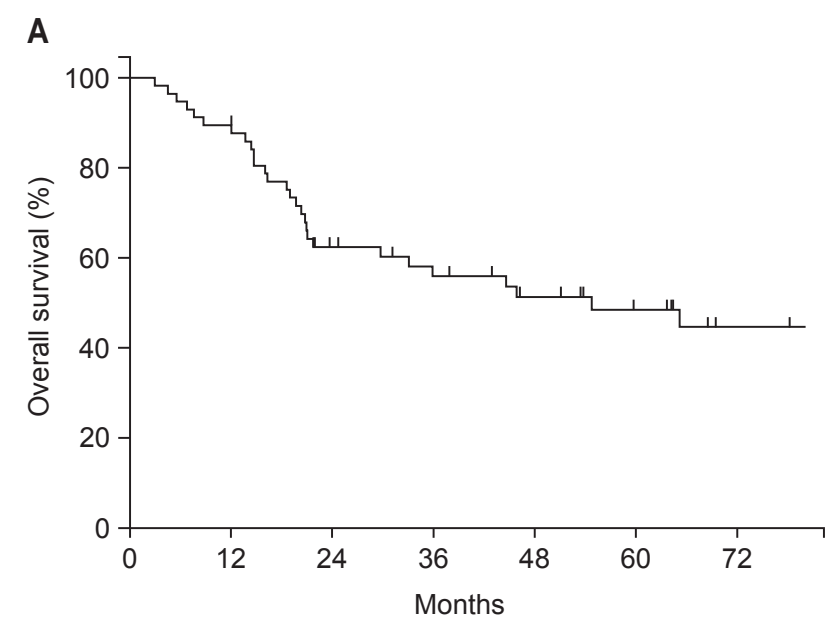

B

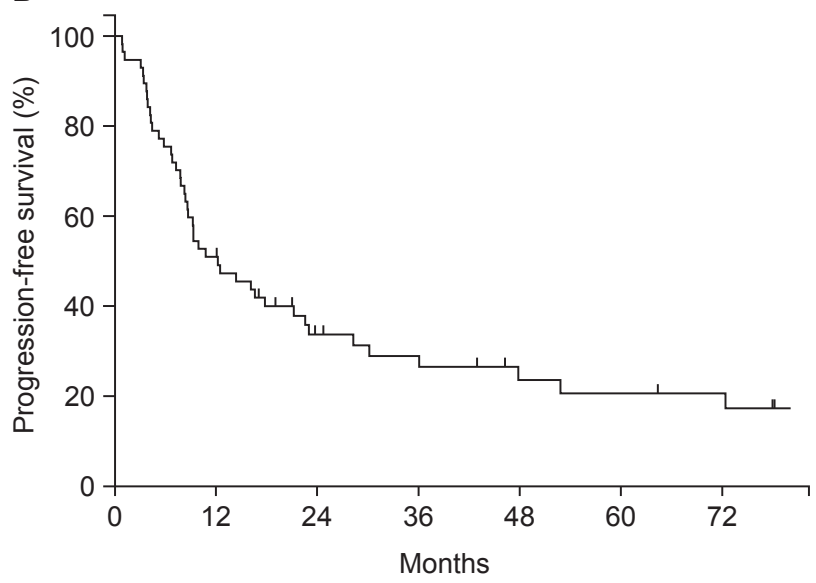

Fig. 1. (A) Overall survival and (B) progression-free survival for the entire patients. 
Table 3. Univariate and multivariate analyses for OS

\begin{tabular}{|c|c|c|c|c|c|c|}
\hline \multirow{2}{*}{ Variable } & \multirow{2}{*}{ No. } & \multicolumn{2}{|c|}{ Univariate } & \multicolumn{3}{|c|}{ Multivariate } \\
\hline & & $2-y r$ OS $(\%)$ & $p$-value & $\mathrm{HR}$ & $95 \% \mathrm{Cl}$ & p-value \\
\hline Age (yr) & & & 0.005 & & & 0.423 \\
\hline$<70$ & 37 & 63.9 & & - & - & \\
\hline$\geq 70$ & 20 & 40.0 & & - & - & \\
\hline Disease-free interval (mo) & & & 0.257 & & & - \\
\hline$\geq 15$ & 27 & 61.0 & & - & - & \\
\hline$<15$ & 30 & 63.3 & & - & - & \\
\hline No. of recurrent foci & & & 0.085 & & & 0.048 \\
\hline 1 & 37 & 70.3 & & 1.000 & - & \\
\hline 2,3 & 20 & 46.7 & & 2.132 & $1.006-4.519$ & \\
\hline Concurrent chemotherapy & & & 0.001 & & & 0.001 \\
\hline No & 15 & 33.3 & & 1.000 & - & \\
\hline Yes & 42 & 73.1 & & 0.303 & $0.147-0.624$ & \\
\hline BED $\left(\mathrm{Gy}_{10}\right)$ & & & 0.704 & & & - \\
\hline$<79.2$ & 19 & 63.2 & & - & - & \\
\hline$\geq 79.2$ & 38 & 62.0 & & - & - & \\
\hline $\mathrm{GTV}\left(\mathrm{cm}^{3}\right)$ & & & 0.989 & & & - \\
\hline$<40$ & 30 & 60.0 & & - & - & \\
\hline$\geq 40$ & 27 & 65.4 & & - & - & \\
\hline Initial tumor response & & & 0.129 & & & 0.108 \\
\hline $\mathrm{CR}$ & 6 & 83.3 & & - & - & \\
\hline$P R, S D, P D$ & 51 & 59.8 & & - & - & \\
\hline
\end{tabular}

OS, overall survival; $\mathrm{HR}$, hazard ratio; $\mathrm{Cl}$, confidence interval; $\mathrm{BED}$, biologically equivalent dose; GTV, gross tumor volume; CR, complete regression; $\mathrm{PR}$, partial regression; $\mathrm{SD}$, stable disease; $\mathrm{PD}$, progressed disease.

A

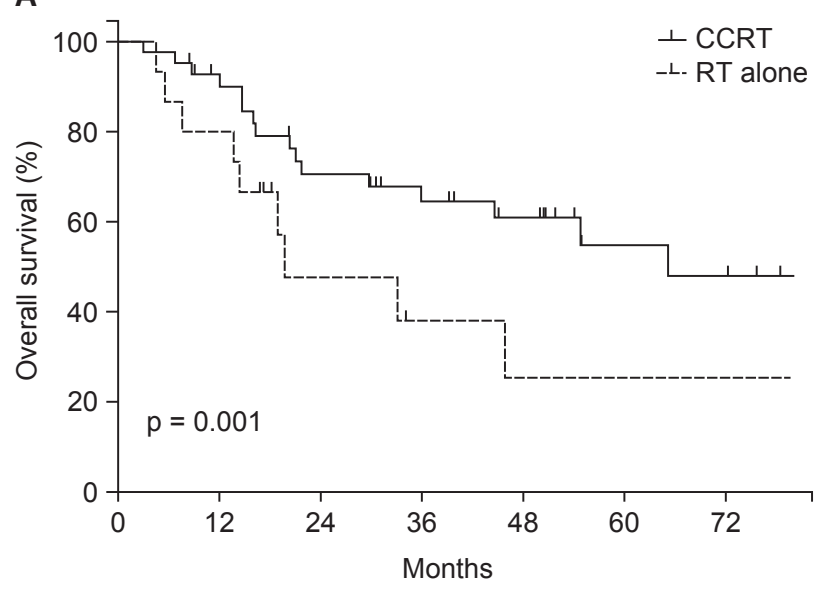

B

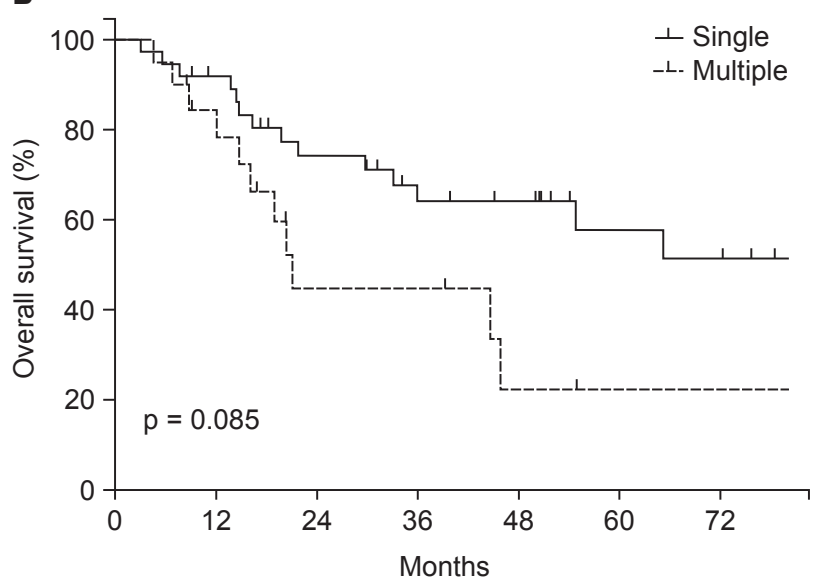

Fig. 2. Overall survival according to (A) the use of concurrent chemotherapy (CCRT) and (B) the number of locoregional recurrent foci.

disease progression over 5 years after salvage treatment. All patients had a single locoregional recurrent focus, and 5 of 7 patients received CCRT.

\section{Toxicity}

The most common side effects were radiation pneumonitis and esophagitis. Acute radiation pneumonitis occurred in 19 patients (33.3\%), with grade 1 in 5 (8.8\%), grade 2 in 11 (19.3\%), and grade 3 in 3 (5.3\%). Esophagitis occurred in 27 patients (47.4\%), with grade 1 in 9 (15.8\%), grade 2 in 16 (28.1\%), and grade 3 in 2 (3.5\%). All acute toxicities were resolved and did not lead to chronic complications. Grade 4-5 toxicities were 
A

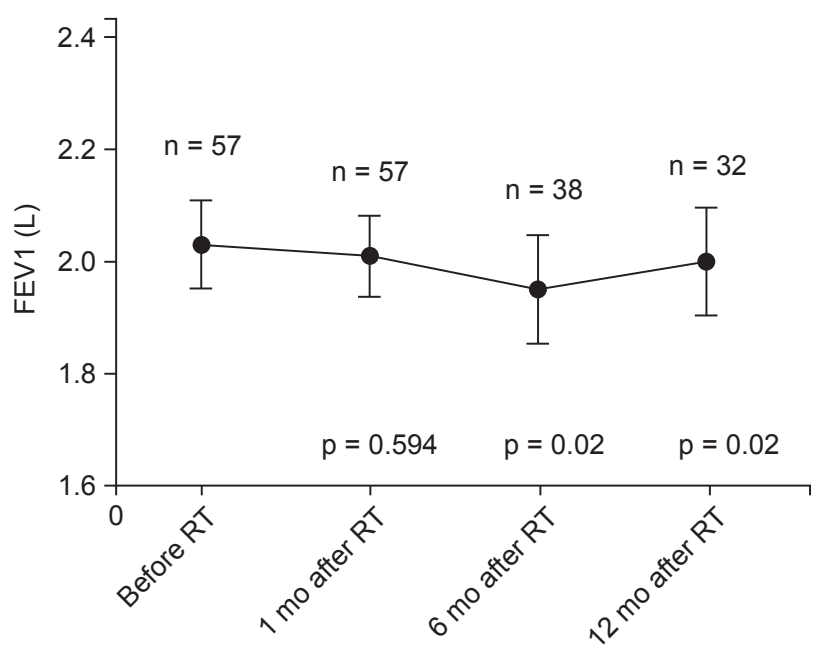

C

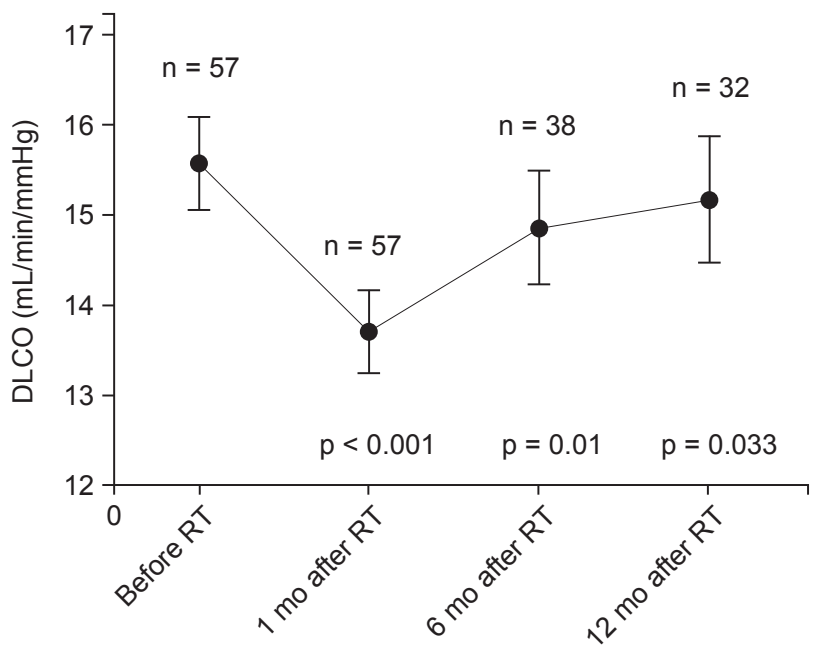

B

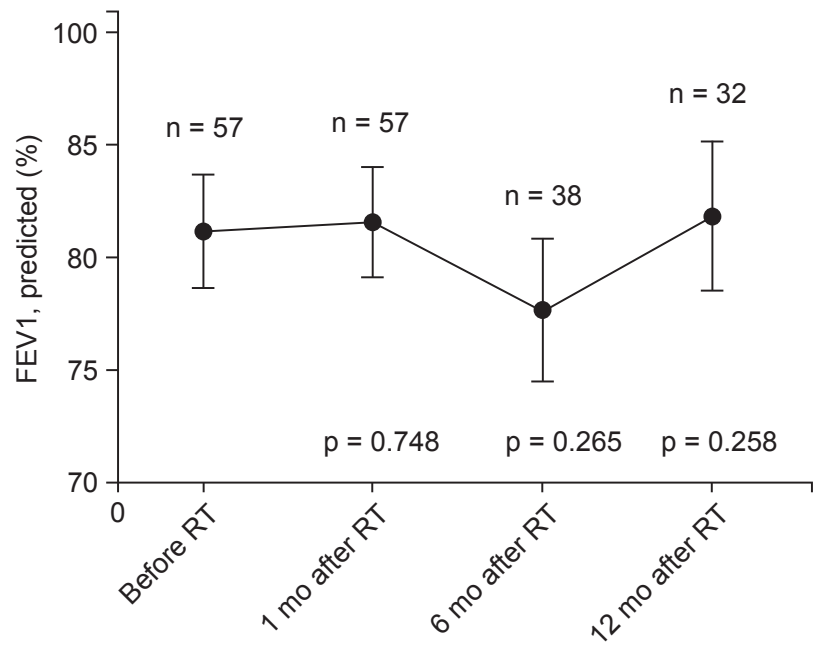

D

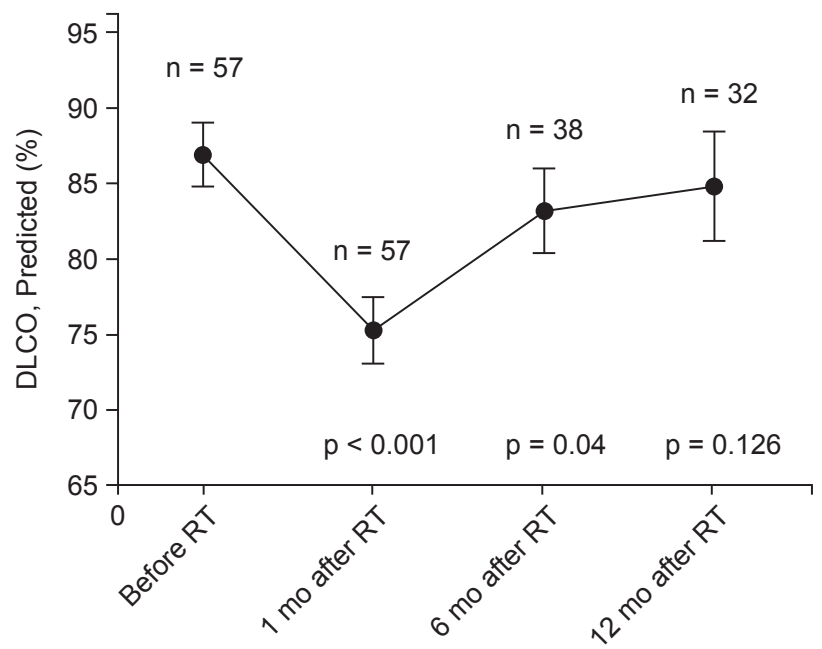

Fig. 3. Change of the (A) forced expiratory volume in 1 second (FEV1), (B) FEV1 predicted, (C) diffusing capacity of the lung for carbon monoxide (DLCO), and (D) DLCO predicted at 1, 6, and 12 months following irradiation. The error bars indicate the standard deviation. RT, radiotherapy; $n$, number of patients; $p$, significance of the paired t-test.

not observed in all patients.

PFT results at baseline and after radiotherapy were shown in Fig. 3. PFT at 1, 6, and 12 months were performed in $100 \%, 66.7 \%$, and $56.1 \%$ of patients, respectively. Before radiotherapy, mean FEV1 \pm SD was $2.03 \pm 0.59 \mathrm{~L}(81.19 \% \pm$ $19.07 \%$ predicted). Mean FEV1 \pm SD at 1, 6, and 12 months after irradiation were $2.01 \pm 0.55 \mathrm{~L}(81.57 \pm 18.47 \%$ predicted $)$, $1.95 \pm 0.6 \mathrm{~L}(77.7 \% \pm 19.53 \%$ predicted $)$, and $2.0 \pm 0.54 \mathrm{~L}$ $(81.86 \% \pm 18.69 \%$ predicted), respectively. Although there was no significant change in FEV1 at 1 month after radiotherapy, it declined at 6 months $(p=0.02)$. However, the change of FEV1 (\% predicted) was not statistically significant. As regard to $\mathrm{DLCO}$, mean DLCO \pm SD was $15.57 \pm 3.85 \mathrm{~mL} / \mathrm{min} / \mathrm{mmHg}$
$(86.92 \% \pm 15.85 \%$ predicted) before radiotherapy. Mean DLCO $\pm \mathrm{SD}$ at 1,6 , and 12 months after radiotherapy were $13.71 \pm$ $3.45 \mathrm{~mL} / \mathrm{min} / \mathrm{mmHg}(75.32 \% \pm 16.46 \%$ predicted $), 14.86 \pm$ $3.86 \mathrm{~mL} / \mathrm{min} / \mathrm{mmHg}(83.22 \% \pm 17.11 \%$ predicted $)$, and $15.17 \pm$ $3.95 \mathrm{~mL} / \mathrm{min} / \mathrm{mmHg}(84.84 \% \pm 20.36 \%$ predicted $)$, respectively. Although DLCO values significantly declined at 1 month after radiotherapy $(p<0.001)$, it improved to the baseline level at 12 months.

\section{Discussion and Conclusion}

This is a retrospective study reporting clinical outcomes of salvage radiotherapy with or without chemotherapy in 
Table 4. Univariate and multivariate analyses for PFS

\begin{tabular}{|c|c|c|c|c|c|c|}
\hline \multirow{2}{*}{ Variable } & \multirow{2}{*}{ No. } & \multicolumn{2}{|c|}{ Univariate } & \multicolumn{3}{|c|}{ Multivariate } \\
\hline & & $2-y r$ PFS (\%) & p-value & $\mathrm{HR}$ & $95 \% \mathrm{Cl}$ & $p$-value \\
\hline Age (yr) & & & 0.019 & & & 0.283 \\
\hline$<70$ & 37 & 41.1 & & - & - & \\
\hline$\geq 70$ & 20 & 20.0 & & - & - & \\
\hline Disease-free interval (mo) & & & 0.604 & & & - \\
\hline$\geq 15$ & 27 & 39.1 & & - & - & \\
\hline$<15$ & 30 & 28.5 & & - & - & \\
\hline No. of recurrent foci & & & 0.010 & & & 0.002 \\
\hline 1 & 37 & 45.2 & & 1.0 & - & \\
\hline 2,3 & 20 & 9.0 & & 2.935 & $1.508-5.714$ & \\
\hline Concurrent chemotherapy & & & 0.065 & & & 0.026 \\
\hline No & 15 & 20.0 & & 1.0 & - & \\
\hline Yes & 42 & 38.2 & & 0.467 & $0.239-0.913$ & \\
\hline BED $\left(\mathrm{Gy}_{10}\right)$ & & & 0.928 & & & - \\
\hline$<79.2$ & 19 & 36.8 & & - & - & \\
\hline$\geq 79.2$ & 38 & 31.8 & & - & - & \\
\hline $\operatorname{GTV}\left(\mathrm{cm}^{3}\right)$ & & & 0.623 & & & - \\
\hline$<40$ & 30 & 36.7 & & - & - & \\
\hline$\geq 40$ & 27 & 29.4 & & - & - & \\
\hline Initial tumor response & & & 0.274 & & & - \\
\hline $\mathrm{CR}$ & 6 & 66.7 & & - & - & \\
\hline$P R, S D, P D$ & 51 & 29.9 & & - & - & \\
\hline
\end{tabular}

PFS, progression-free survival; $\mathrm{HR}$, hazard ratio; $\mathrm{Cl}$, confidence interval; $\mathrm{BED}$, biologically equivalent dose; GTV, gross tumor volume; CR, complete regression; PR, partial regression; SD, stable disease; PD, progressed disease.

locoregionally recurrent NSCLC patients after radical surgery. In our study, salvage radiotherapy showed promising survival outcomes. Median OS and PFS were 54.8 months and 12.2 months, respectively, and these were significantly associated with concurrent chemotherapy and single recurrence focus. Although we observed a significant decrease in DLCO 1 month after radiotherapy, we did not observe the decrease in FEV1 and any chronic pulmonary complications.

Until now, there is no standard treatment for locoregional recurrent NSCLC after curative surgery. Several retrospective studies evaluated survival of patients with postoperative recurrent NSCLC in modern era (Table 5). They reported that 2-year and median OS have ranged from 30\% to 60\% and 14 months to 31 months, respectively [7-13]. Our survival outcome is better than previous studies. This good clinical outcome in the present study could have resulted from the accurate re-staging with PET-CT, favorable patients with initially early-stage disease, good PS, and limited recurrent focus, the use of concurrent chemotherapy, and the adequate radiation dose in modern era.

The role of concurrent chemotherapy in post-resection recurrent NSCLC has not been well studied. For non-metastatic inoperable NSCLC patients, the European Organisation for Research and Treatment of Cancer (EORTC) performed a phase III randomized trial comparing cisplatin-based CCRT with radiotherapy [16]. The study demonstrated that survival was significantly improved with CCRT. However, this clinical benefit came at the price of substantial side effects such as nausea, vomiting, and severe esophagitis. A systematic review evaluating radical CCRT also reported that CCRT significantly improved OS and PFS with the increased acute toxicity [17]. In the salvage setting, Bar et al. [11] reported clinical outcomes of 30 locoregional recurrent patients treated with CCRT. They used chemotherapy regimen as follows: platinum/vinorelbine (47\%), carboplatin/paclitaxel (23\%), cisplatin/etoposide (13\%), and other platinum agents (17\%). The median survival after recurrence was 26.9 months. Grade 4 toxicity occurred in 7 patients; 1 radiation pneumonitis, 1 pulmonary emboli, 5 febrile neutropenia. There was no severe radiation esophagitis. Takenaka et al. [12] also analyzed the effect of CCRT for recurrent NSCLC. The median PFS and OS were 13 months (range, 4 to 127 months) and 31 months (range, 5 to 127 months), respectively. Chemotherapy included a platinum agent in all cases: cisplatin-based CCRT in 23, carboplatin- 


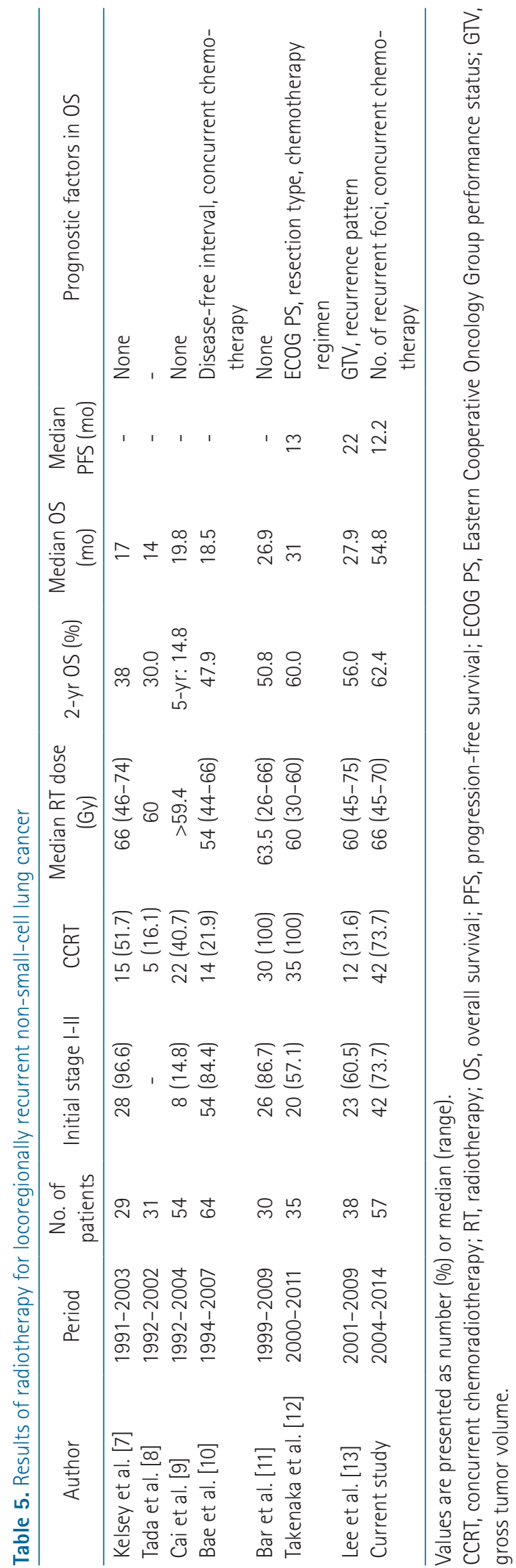

based CCRT in 12. Grade 3 esophagitis occurred in 3 patients (9\%), and grade 4 hematologic toxicity occurred in 7 patients (20\%). In our study, 42 patients were concurrently treated with weekly paclitaxel/cisplatin $(n=40)$ or triweekly etoposide/ cisplatin $(n=2)$. Five patients experienced acute grade 3 toxicities, and chronic complications did not occur in our cohort. These results suggest that side effects of CCRT are tolerable in postoperative recurrent NSCLC patients.

Bae et al. [10] reported the survival outcomes of high dose salvage radiotherapy for NSCLC patients with locoregional recurrence after resection. The median total $\mathrm{BED}_{10}$ was $70.2 \mathrm{~Gy}_{10}$ (range, 51.5 to $85.8 \mathrm{~Gy}_{10}$ ). There was a significant difference in the 2-year survival rates between BED $\leq 70.2 \mathrm{~Gy}_{10}$ and $>70.2 \mathrm{~Gy}_{10}$ groups (37.3\% vs. $59.2 \%, \mathrm{p}=0.025$ ). In the current study, the median BED was $79.2 \mathrm{~Gy}_{10}$ (range, 58.5 to 84 $\left.\mathrm{Gy}_{10}\right)$, and most patients except in two received radiotherapy higher than BED $70 \mathrm{~Gy}_{10}$. From our data, we could not find survival difference between BED $<79.2 \mathrm{~Gy}_{10}$ and $\geq 79.2 \mathrm{~Gy}_{10}$ groups. It suggests that increasing radiation dose does not improve survival when the radiation dose is adequate with a conformal technique.

In our investigation, all patients were evaluated with PET$\mathrm{CT}$ before radiotherapy. It is valuable for accurate re-staging of the mediastinal lymph nodes. Although PET has a tendency to overestimate the disease extent, it could contribute to improve clinical outcomes and help to select appropriate patients for local therapy [18]. We delineated the target volume based on PET images, and elective nodal irradiation was omitted. In this study, 39 patients (68.4\%) showed treatment failure during follow-up period. Isolated in-field recurrence was reported in 5 patients (8.8\%). Kelsey et al. [7] used elective nodal irradiation in 27 of 29 NSCLC patients in salvage radiotherapy. They reported that $34.4 \%$ of patients showed failure within the radiation field despite elective nodal irradiation. Our locoregional recurrence rate $(n=17,29.8 \%)$ is not different from elective nodal irradiated cases. In addition, distant metastasis ( $n=31,54.4 \%)$ was more dominant than locoregional recurrence in our study. Therefore, we suggest that omitting elective nodal irradiation does not result in poor clinical outcomes as salvage radiotherapy.

The prognostic factors for survival of recurrent NSCLC patients treated with salvage radiotherapy have been proposed by other investigators. PS, resection type, disease-free interval, CCRT, concurrent chemotherapy regimen, recurrence pattern, and GTV have been reported as prognostic factors for OS $[9,10,12,13]$. In the present study, disease-free interval, PS, type of operation, and GTV volume did not show significant 
association with survival. We found that a single recurrent focus and CCRT significantly improved OS and PFS.

Postoperative radiotherapy could give an additional harm to patients who had already compromised lung function after surgery. In this study, we attempted to analyze the changes in PFTs in locoregional recurrent NSCLC patients treated with salvage radiotherapy. Our study revealed that patients receiving radiotherapy experienced a significant decrease in DLCO. Although DLCO declined significantly, grade 3 acute pneumonitis occurred in 3 patients (5.3\%). In addition, previous studies did not report any severe complications. Based on these findings, it is thought that salvage radiotherapy with conformal technique is a safe treatment modality for recurrent NSCLC after radical resection.

This study had several limitations. First, this is a single institutional retrospective study. Intrinsic bias may have not been removed due to this nature. Second, included patient number was small and may restrict statistical power. Prospective studies with a large sample size are needed.

In summary, the present study indicated that salvage radiotherapy produced good survival outcomes in patients with recurrent NSCLC after surgery in the modern era. Although patients experienced a significant decline in DLCO immediately after radiotherapy, it recovered to pretreatment level within 12 months, and no one experienced significant chronic complications. In addition, a single recurrent focus and the use of concurrent chemotherapy were independent favorable prognostic factors for OS and PFS. These findings suggested that salvage radiotherapy combined with chemotherapy could be a promising therapy for selected patients with locoregional recurrence of $\mathrm{NSCLC}$.

\section{Conflict of Interest}

No potential conflict of interest relevant to this article was reported.

\section{References}

1. Oh CM, Won YJ, Jung KW, et al. Cancer statistics in korea: incidence, mortality, survival, and prevalence in 2013. Cancer Res Treat 2016;48:436-50.

2. Sugimura $H$, Nichols FC, Yang $P$, et al. Survival after recurrent nonsmall-cell lung cancer after complete pulmonary resection. Ann Thorac Surg 2007;83:409-17.

3. Hung JJ, Hsu WH, Hsieh CC, et al. Post-recurrence survival in completely resected stage I non-small cell lung cancer with local recurrence. Thorax 2009;64:192-6.

4. Endo C, Sakurada A, Notsuda H, et al. Results of long-term follow-up of patients with completely resected non-small cell lung cancer. Ann Thorac Surg 2012;93:1061-8.

5. Voltolini L, Paladini P, Luzzi L, Ghiribelli C, Di Bisceglie M, Gotti G. Iterative surgical resections for local recurrent and second primary bronchogenic carcinoma. Eur J Cardiothorac Surg 2000;18:529-34.

6. National Comprehensive Cancer Network. NCCN clinical practice guidelines in oncology (NCCN Guidelines): non-small cell lung cancer. v4. 2016 [Internet]. Fort Washington, PA: National Comprehensive Cancer Network; c2016. [cited 2016 July 20]. Available from: http://www.nccn.org/professionals/ physician_gls/pdf/nsclc.pdf.

7. Kelsey CR, Clough RW, Marks LB. Local recurrence following initial resection of NSCLC: salvage is possible with radiation therapy. Cancer J 2006;12:283-8.

8. Tada T, Fukuda H, Nakagawa K, et al. Non-small cell lung cancer: radiation therapy for locoregional recurrence after complete resection. Int J Clin Oncol 2005;10:425-8.

9. Cai $X W, X u L Y$, Wang $L$, et al. Comparative survival in patients with postresection recurrent versus newly diagnosed nonsmall-cell lung cancer treated with radiotherapy. Int J Radiat Oncol Biol Phys 2010;76:1100-5.

10. Bae SH, Ahn YC, Nam H, et al. High dose involved field radiation therapy as salvage for loco-regional recurrence of non-small cell lung cancer. Yonsei Med J 2012;53:1120-7.

11. Bar J, Ng D, Moretto P, et al. Chemoradiotherapy for locoregional recurrence of non-small-cell lung cancer after surgical resection: a retrospective analysis. Clin Lung Cancer 2013;14:200-4.

12. Takenaka T, Takenoyama M, Toyozawa R, et al. Concurrent chemoradiotherapy for patients with postoperative recurrence of surgically resected non-small-cell lung cancer. Clin Lung Cancer 2015;16:51-6.

13. Lee NK, Moon SH, Kim TH, et al. Prognostic value of gross tumor volume for definitive radiation therapy in patients with locoregionally recurrent non-small-cell lung cancer after surgical resection. Clin Lung Cancer 2013;14:399-406.

14. Borst GR, De Jaeger $K$, Belderbos JS, Burgers SA, Lebesque JV. Pulmonary function changes after radiotherapy in non-smallcell lung cancer patients with long-term disease-free survival. Int J Radiat Oncol Biol Phys 2005;62:639-44.

15. Dautzenberg $B$, Arriagada $R$, Chammard $A B$, et al. A controlled study of postoperative radiotherapy for patients with completely resected nonsmall cell lung carcinoma. Groupe d'Etude et de Traitement des Cancers Bronchiques. Cancer 1999:86:265-73.

16. Schaake-Koning C, van den Bogaert W, Dalesio O, et al. Effects of concomitant cisplatin and radiotherapy on inoperable nonsmall-cell lung cancer. N Engl J Med 1992;326:524-30. 
17. O'Rourke N, Roque I Figuls M, Farre Bernado N, Macbeth F. Concurrent chemoradiotherapy in non-small cell lung cancer. Cochrane Database Syst Rev 2010:CD002140.

18. Bille A, Pelosi $E$, Skanjeti $A$, et al. Preoperative intrathoracic lymph node staging in patients with non-small-cell lung cancer: accuracy of integrated positron emission tomography and computed tomography. Eur J Cardiothorac Surg 2009;36:440-5. 